\title{
Clinical Features and Management of Patients with an Anoma- lous Origin of the Coronary Arteries
}

\author{
Carolina Espejo-Paeres*, Carmen Olmos, Pedro Martínez-Losas, Fabián Islas, \\ Javier Higueras-Nafria, David Vivas and Isidre Vilacosta
}

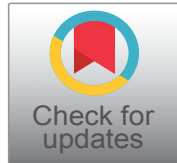

Cardiovascular Institute, Hospital Clínico San Carlos, Universidad Complutense, Spain

*Corresponding author: Carolina Espejo-Paeres, MD, Cardiovascular Institute, Hospital Clínico San Carlos, Universidad Complutense, c/Profesor Martín Lagos s/n 28040 Madrid, Spain, Tel: +34-91-330-30-00-01, Fax: +34-91-330-31-82,

E-mail: carolina.espejo.paeres@gmail.com

\begin{abstract}
Background: An anomalous origin of a coronary artery is a rare congenital condition in which a coronary artery arises from the opposite coronary sinus. Most coronary artery anomalies are an incidental finding without clinical significance, although some cases may be potentially life threatening, depending on the origin, course and termination of the anomalous vessel. Anatomical features of risk are an aortic intramural course or inter-arterial course.

Methods: We present 4 cases of an anomalous origin of the coronary arteries with radiological confirmation by cardiac computed tomography angiography (CCTA) and clinical history of chest pain. Two patients were incidentally diagnosed by invasive coronary angiography, while two of them underwent a CCTA exam directly. Ischemia detection tests such as treadmill test or myocardial perfusion imaging with single photon emission computed tomography (SPECT) were performed in all of them to rule out ischemia.

Results: In all 4 cases, none relationship between clinical symptoms and anatomical features was established. Once ischemia was ruled-out by ischemia detection tests, we chose clinical follow-up instead of surgery given the absence of anatomical or risk clinical features.

Conclusion: Only a minority of patients have symptoms due to these coronary anomalies. An expectant attitude with periodical surveillance seems to be the best option in patients with low-risk variants of coronary artery anomalies without clinical documentation of myocardial ischemia.
\end{abstract}

\section{Introduction}

An anomalous origin of a coronary artery is a rare congenital condition that affects $1 \%$ of the population $[1,2]$. It

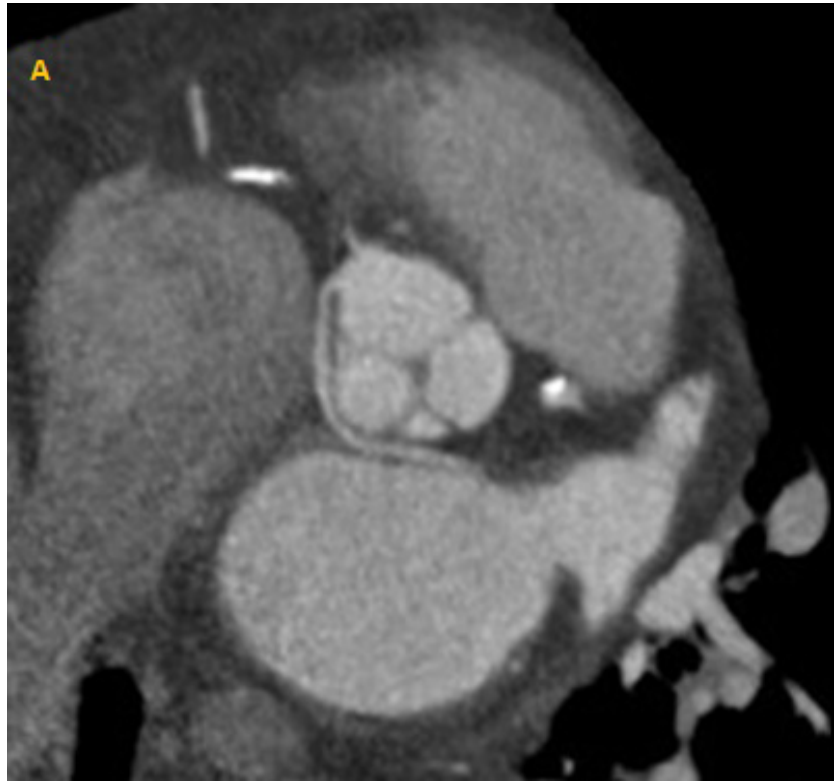

Figure 1: Coronary computed tomography angiography example of anomalous origin of the coronary arteries: Left coronary artery originating from the right coronary sinus, with a retro-aortic course.

is an anatomical variation in which a coronary artery arises from the opposite coronary sinus (Figure 1 and Figure 2). Some authors have described the anomalous origin of the right coronary artery (RCA) from the left coronary sinus (66\% cases) as the most frequent coronary anomaly $[1,3]$. However, other case series have reported an anomalous origin of the circumflex $(\mathrm{Cx})$ artery from the right coronary sinus with a retro-aortic course as the more prevalent [2].

Citation: Espejo-Paeres C, Olmos C, Martínez-Losas P, Islas F, Higueras-Nafria J, et al. (2018) Clinical Features and Management of Patients with an Anomalous Origin of the Coronary Arteries. Int Arch Cardiovasc Dis 2:005

Accepted: May 28, 2018; Published: May 30, 2018

Copyright: (C) 2018 Espejo-Paeres C, et al. This is an open-access article distributed under the terms of the Creative Commons Attribution License, which permits unrestricted use, distribution, and reproduction in any medium, provided the original author and source are credited. 
The anomalous origin of the artery is usually identified while patients undergo invasive coronary angiography or computed tomography angiography (CTA) due to chest pain $[2,3]$. Most coronary artery anomalies are an incidental finding without clinical significance, although some cases may be potentially life threatening, depending on the origin, course and termination of the anomalous vessel [1-3]. Thus, a coronary anomaly can cause sudden death due to ischemia in young, previously asymptomatic subjects with anatomical features of risk: Aortic intramural course or inter-arterial course between the aorta and the pulmonary artery [1,2]. Hence, an accurate recognition of the anomalous aortic origin of a coronary artery is essential to identify those patients at risk [3].

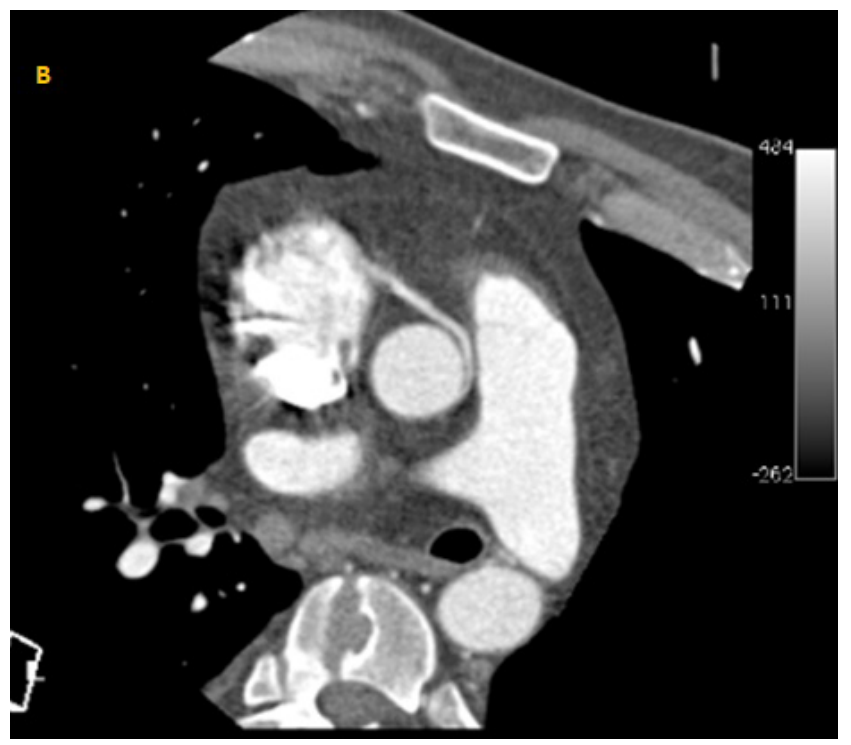

Figure 2: Coronary computed tomography angiography example of anomalous origin of the coronary arteries: Anomalous origin of the right coronary artery from the left coronary sinus, with an inter-arterial course.
CTA is able to diagnose the anomaly and provides better images of the anatomic course compared to invasive coronary angiography. Therefore, it may give prognostic information detecting high-risk variants and it is considered the gold standard for this purpose with a Class I recommendation $[4,5]$.

\section{Methods and Results}

We present 4 cases of an anomalous origin of the coronary artery with radiological confirmation by CTA.

The first case was a 55-year-old male with chest discomfort at rest for the last month and a single episode of syncope after performing aerobic exercise. Cardiac enzymes were normal and the electrocardiogram (ECG) showed an inferior $Q$ wave. The diagnostic work-up included a treadmill test and a SPECT, without identifying myocardial ischemia. Due to persistence of symptoms, an invasive coronary angiography was performed showing an anomalous origin of the $\mathrm{Cx}$ artery from the right sinus. Following the current guidelines recommendation, a CTA was done to accurately document the coronary artery course. A retro-aortic trajectory of the anomalous segment was observed.

The second case was a 35-year-old man with chest pain at rest for five days, with normal biomarkers and a left bundle branch block on the ECG. CTA evidenced an anomalous origin of the RCA from the left sinus with an inter-arterial route between the aorta and the main pulmonary artery, with minor narrowing of the artery but a good distal calibre. Cardiac SPECT did not show any myocardial perfusion abnormality and no relationship was found between the coronary anomaly and clinical symptoms (Figure $3 a$ and Figure $3 b$ ).

The third patient was a 40-year-old male referred to our hospital because of syncope and atypical chest pain for the last 3 months. Resting ECG, treadmill test

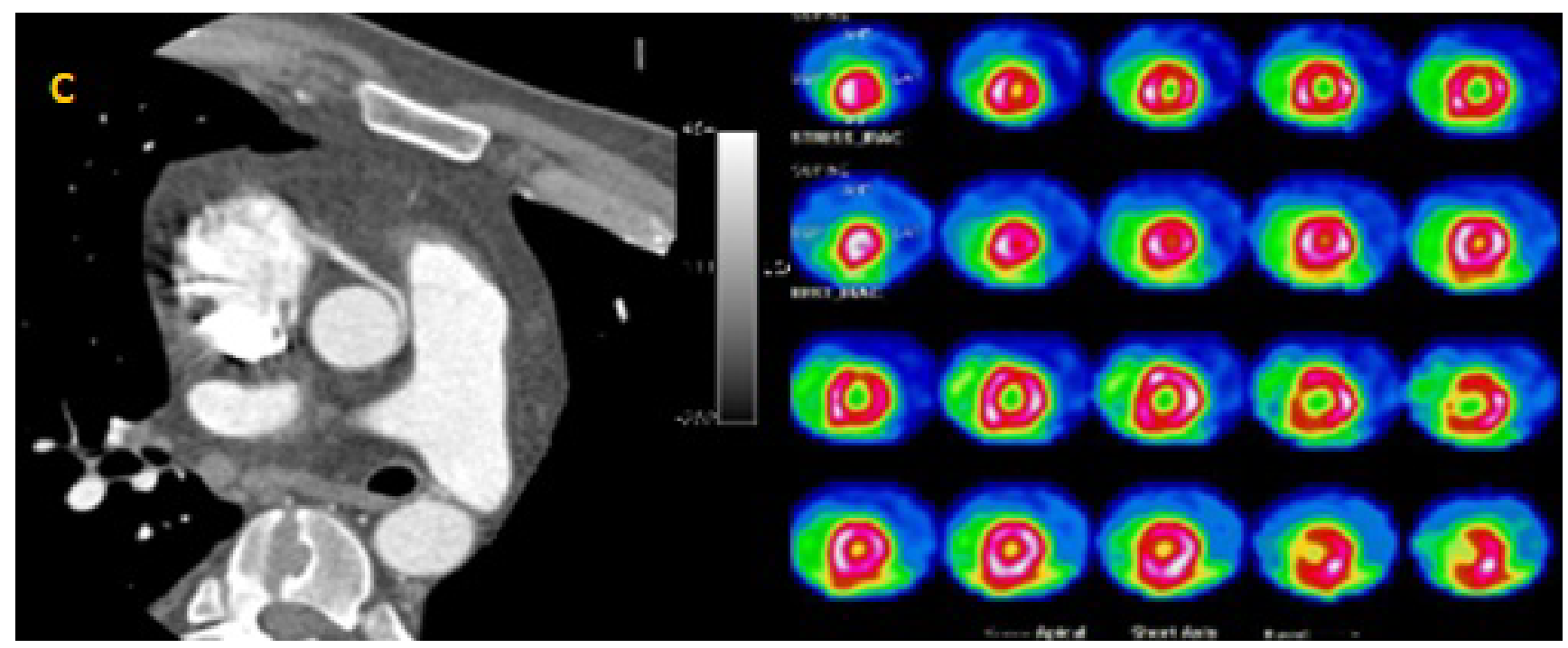

Figure 3: Diagnostic work-up of a patient with an anomalous origin of the right coronary artery (Case 2). A) Computed tomography angiography. The right coronary artery arises from the left coronary sinus, with an inter-arterial course B) Myocardial perfusion SPECT negative for ischemia. 


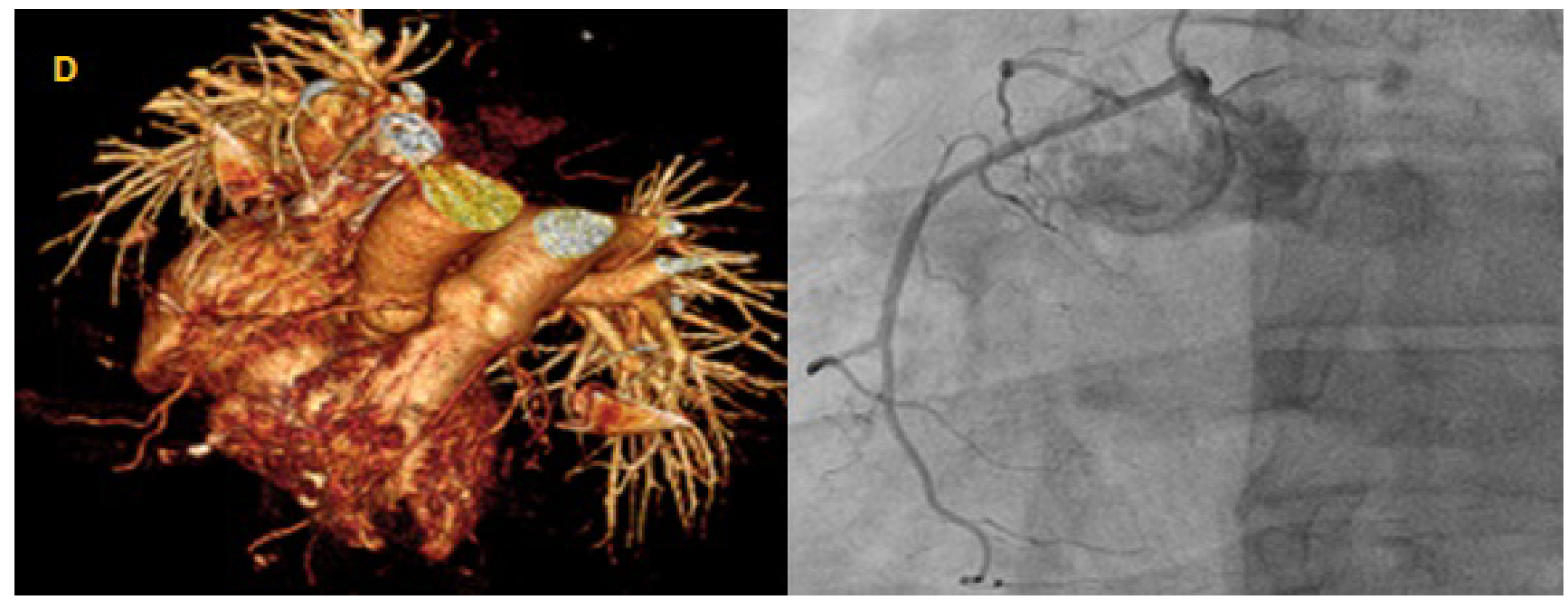

Figure 4: Diagnostic work-up of a patient with an anomalous origin of the right coronary artery (Case 3). A) Three-dimensional volume-rendered image from a computed tomography angiography. The right coronary artery origin arises from the left coronary sinus, and crosses anterior to the aorta, showing no inter-arterial route; B) Invasive coronary angiography (LAO $30^{\circ}$ Cranial $20^{\circ}$ ). The right coronary artery is originating from the left coronary sinus and crossing anterior to the aorta.

Table 1: Clinical and anatomical features of 4 cases with anomalous origin of a coronary artery and chest pain.

\begin{tabular}{|l|l|l|l|l|l|}
\hline $\mathbf{n = 4}$ & Age (years-old) & Symptoms & CTA & $\begin{array}{l}\text { Invasive coronary } \\
\text { angiography } \\
\text { performed? }\end{array}$ & $\begin{array}{l}\text { Ischemia detection } \\
\text { test }\end{array}$ \\
\hline Case 1 & 55 & Chest pain, syncope & $\begin{array}{l}\text { Cx from right sinus } \\
\text { Retro-aortic course }\end{array}$ & Yes & Negative result \\
\hline Case 2 & 35 & Chest pain & $\begin{array}{l}\text { RCA from left sinus } \\
\text { Inter-arterial course }\end{array}$ & No & Negative result \\
\hline Case 3 & 40 & Chest pain, syncope & $\begin{array}{l}\text { RCA from left sinus } \\
\text { Pre-aortic course } \\
\text { Cx from right sinus } \\
\text { Retro-aortic course }\end{array}$ & Yes & Yes \\
\hline Case 4 & 65 & Chest pain & Negative result & \\
\hline
\end{tabular}

CTA: computed tomography angiography; Cx: circumflex artery; RCA: right coronary artery.

and myocardial perfusion imaging with SPECT were completely normal. By CTA, an anomalous origin of the RCA arising from the left sinus with a pre-aortic course (without risk features) was confirmed. The patient had previously been studied in another centre because of chest pain and a coronary angiography had been performed. The CTA allowed to more accurately define the RCA course and to rule out high-risk features (Figure $4 a$ and Figure $4 b$ ).

The last patient was a 65 -year-old man who was admitted to hospital because of a first episode of heart failure, atypical chest pain and slight elevation of cardiac biomarkers. Invasive coronary angiography showed a chronic occlusion of the RCA. In addition, the study also showed a $C x$ artery arising from the right sinus without risk variants. Cardiac SPECT demonstrated an inferior fixed perfusion defect, concordant with the chronic occlusion.

To sum up, two of the four patients had a history of atypical chest pain and episodes of syncope, while the other two patients had isolated chest pain. In two cases the anomalous origin was incidentally diagnosed by invasive coronary angiography during work-up for chest pain (with subsequent CTA); The other two patients underwent a CTA exam directly. Regarding ischemia detection tests, a treadmill test or a SPECT were performed in all of them to rule out ischemia (Table 1).

In all 4 cases, none relationship between clinical symptoms and anatomical features was established. Once high-risk anatomic features were excluded by CTA and ischemia was ruled-out by ischemia detection tests in all cases, we chose clinical follow-up instead of surgery. In the 4 patients, follow-up was performed in the outpatient clinic, with thorough clinical history, physical exam, and rest ECG, and one year after discharge all patients remained asymptomatic.

\section{Discussion}

An anomalous aortic origin of a coronary artery is a rare congenital condition in which the left coronary artery (LCA) arises from the right sinus of Valsalva or RCA arises from the left sinus of Valsalva [1,2].

This rare defect can cause chest pain, syncope or sudden death, (typically during exercise, triggered by myocardial ischemia). Myocardial ischemia may be present due to mechanical compression of the anomalous ar- 
tery by the great vessels or due to a slit-like orifice in the aortic wall. High-risk features for sudden death are: Origin with a narrow orifice, an acute angle relative to the aorta, an aortic intramural course or an inter-arterial course of the anomalous segment between the aorta and the main pulmonary artery $[2,3]$.

The most common variant is the origin of the RCA from the left coronary sinus $[1,3]$ and the one associated with the highest risk is a left main originated from the right coronary sinus (60\% have an inter-arterial course). The cases with an aortic intramural course and/or an inter-arterial course entail more risk of adverse events, due to a higher probability of ischaemia than those cases without these findings. A LCA arising from the right sinus of Valsalva, is associated to higher risk to $S C D$, since it most frequently has an intramural or inter-arterial course. On the other hand, a RCA arising from the left sinus involves less risk because it less frequently has an intramural or inter-arterial course [3].

Many patients with abnormal coronary arteries remain asymptomatic most of their lives, and the anomaly is usually an incidental finding after coronary angiography during the study of chest pain $[1,2]$.

If myocardial ischemia is documented, or in patients with high-risk anatomical features, there are two treatment options: Percutaneous coronary intervention and stenting or surgical repair [3]. Surgical choice has a Class I recommendation in patients with radiological and clinical risk features [5]. Nevertheless, the longterm benefits of such therapies have not been demonstrated among patients older than 35 years without documented ischemia [3]. The prognosis in these middle-aged-to-elderly patients is good despite the lack of surgical treatment, as sudden death mostly occurs before 35 years of age. In such cases, the objective is adequate symptomatic relief $[3,6]$. In a five-year follow-up study that included patients with congenital coronary anomalies managed medically, no death was found to be directly related to the congenital defect [6]. Considering previous data and the fact that our patients were 35 years old or older and ischemia was not established by specific tests, we chose a conservative approach with medical follow-up.
As a conclusion, an expectant attitude with periodical surveillance seems to be the best option in patients with low-risk variants of coronary artery anomalies, without clinical expression or documentation of myocardial ischemia.

Only a minority of patients has symptoms due to these coronary anomalies, so the surgical decision is controversial and should be based on anatomical features and ischemia demonstration.

\section{Declaration of Interest}

The authors report no relationships that could be construed as a conflict of interest. The authors alone are responsible for the content and writing of the paper.

\section{References}

1. Cho SH, Joo HC, Yoo KJ, Youn YN (2015) Anomalous origin of right coronary artery from left coronary sinus: Surgical management and clinical result. Thorac Cardiovasc Surg 63: 360-366.

2. Szymczyk K, Polguj M, Szymczyk E, Majos A, Grzelak $P$, et al. (2014) Prevalence of congenital coronary artery anomalies and variants in 726 consecutive patients based on 64-slice coronary computed tomography angiography. Folia Morphol (Warsz) 73: 51-57.

3. Dubey $L$ (2013) Anomalous origin of the coronary arteries: An account of six cases. JNMA J Nepal Med Assoc 52: 512-516.

4. Krupiński M, Urbańczyk-Zawadzka M, Laskowicz B, Irzyk M, Banyś R, et al. (2014) Anomalous origin of the coronary artery from the wrong coronary sinus evaluated with computed tomography: "High-risk" anatomy and its clinical relevance. Eur Radiol 24: 2353-2359.

5. Warnes CA, Williams RG, Bashore TM, Child JS, Connolly $\mathrm{HM}$, et al. (2008) ACC/AHA 2008 guidelines for the management of adults with congenital heart disease: A report of the american college of cardiology/american heart association task force on practice guidelines (writing committee to develop guidelines on the management of adults with congenital heart disease). Developed in collaboration with the american society of echocardiography, heart rhythm society, international society for adult congenital heart disease, society for cardiovascular angiography and interventions, and society of thoracic surgeons. J Am Coll Cardiol 52: 143-263.

6. Kaku B, Shimizu M, Yoshio $H$, Ino $H$, Mizuno $S$, et al. (1996) Clinical features of prognosis of japanese patients with anomalous origin of the coronary artery. Jpn Circ J 60: 731-741. 